\title{
Medida de Calor Específico e Lei de Resfriamento de Newton: Um Refinamento na Análise dos Dados Experimentais
}

\author{
(Measurement of Specific Heat and Newton's Law of Cooling: A Refinement in the Analysis of the Experimental Data) \\ Wilton Pereira da Silva,, Jürgen W. Precker, Cleide M. D. P. S. e Silva, \\ Diogo D. P. S. e Silva e Cleiton D. P. S. e Silva \\ Grupo de Aquisição e Tratamento de Dados, Departamento de Física \\ Universidade Federal de Campina Grande, Campina Grande, 58109-970, PB, Brasil
}

Recebido em 05 de junho, 2003. Manuscrito revisado em 03 de setembro, 2003. Aceito em 24 de novembro, 2003.

\begin{abstract}
Uma das técnicas para a determinação do calor específico de sólidos e líquidos dispensa o uso de um calorímetro e utiliza a lei de resfriamento de Newton na análise dos dados experimentais. O sucesso desta técnica pressupõe que seja possível determinar, com pequenas incertezas, as temperaturas do sistema imediatamente antes e imediatamente depois das transferências internas de calor devido à imersão do corpo (cujo calor específico se deseja medir) em um recipiente contendo água quente. Neste artigo é proposto um refinamento desta técnica utilizando-se dois ajustes de curvas para a função que descreve a lei de resfriamento, com pequenas extrapolações, para se determinar essas temperaturas e também as suas incertezas. Isto possibilita determinar não só o valor médio do calor específico, mas também a incerteza deste valor médio, por propagação de erros. O ajuste da própria função que descreve a lei de resfriamento aos dados possibilitou a comparação imediata dos parâmetros obtidos experimentalmente com aqueles previstos pela teoria, e há uma grande concordância entre eles. A aplicação deste refinamento na determinação do calor específico do alumínio indica que tal procedimento é eficaz mesmo quando se trabalha com instrumentos de baixo custo, voltados para o ensino.
\end{abstract}

\begin{abstract}
One of the techniques for the determination of the specific heat of solids and liquids use the Newton's law of cooling for the analysis of the experimental data instead of the usual calorimeter method. The success of this technique depends on the possibility in determining the temperatures of the system immediately before and after the internal heat transfer due to the immersion of the sample (whose specific heat one wants to measure) with small uncertainties in a container filled with hot water. In this paper a refinement of this technique is proposed using two curve fittings for the function that describes the law of cooling and small extrapolations to determine those temperatures, as well their uncertainties. This allows to determine not only the value of the specific heat, but also the uncertainty of this value by error propagation. The fitting of the function that describes the law of cooling (and not of polynomials) to the data allows the comparison of the parameters obtained from the experiment with those predicted by the theory, and there is good agreement. The application of this refinement to the determination of the specific heat of aluminium indicates that the procedure is good in spite of the use of low cost apparatus.
\end{abstract}

\section{Introdução}

No estudo de termodinâmica, um conceito útil para quantificar uma certa quantidade de calor transferida a um sistema é o conceito de calor específico. Uma definição simples pode ser dada assim: o calor específico é a quantidade de calor que deve ser transferida a $1 \mathrm{~g}$ de uma substância para que a sua temperatura seja elevada em $1^{0} \mathrm{C}$. Conforme se sabe, essa quantidade de calor varia de substância para substância e, então, o calor específico é um parâmetro que caracteriza uma dada substância. Existem diversas técnicas para a medição do calor específico de uma substância e, aqui, serão mencionadas duas delas, normalmente utilizadas para líquidos e sólidos.

A primeira técnica utiliza um calorímetro (neste artigo entendido como um recipiente de paredes quase adiabáticas) contendo água, numa temperatura conhecida, e neste sistema é introduzido, numa dada temperatura, um corpo feito da substância cujo calor específico se deseja medir. Após a medição da temperatura de equilíbrio do sistema, um balanço nas transferências de calor possibilita determinar o calor especifico da substância do corpo imerso. Este experimento é descrito em praticamente todos os livros que tratam de termodinâmica básica. O problema experimental é proposto, por exemplo, na Ref. [1] e o valor do calor específico da substância investigada é obtido utilizando-se a equação

*wiltonps@uol.com.br 
que dá o balanço de calor: "o calor absorvido é igual ao calor cedido". Alguns destes livros observam, entretanto, que parte do calor disponível é transferido para o meio ambiente, o que acarreta erros experimentais. Mesmo com erros, uma vez conhecida uma estimativa razoável do calor específico, o experimento poderia ser refeito impondo-se a condição de que o sistema água/calorímetro tenha uma temperatura previamente determinada, abaixo da temperatura ambiente, de forma a compensar perdas na parte final do processo [2]. Uma pesquisa bibliográfica mais minuciosa indica que existem vários arranjos engenhosos para considerar perdas de calor em um calorímetro usado na medição do calor específico de uma substância, e um desses arranjos é proposto em [3]. Já uma discussão sobre a determinação de calor específico utilizando a aproximação de que não haja perdas de energia num certo tipo de calorímetro, constituído de uma garrafa de aço com tampa de PVC, é apresentada em [4].

Uma segunda técnica de medição do calor específico de um corpo não usa calorímetro, e parte do pressuposto de que o sistema vai perder calor continuamente para o meio ambiente, levando esse fato em consideração. Para usar esta técnica é necessário conhecer "como" o sistema perde calor para o meio ambiente antes e após a imersão do corpo investigado, o que é possível através da aplicação da "lei de resfriamento (ou esfriamento) de Newton”. A idéia básica é utilizar um recipiente com água, numa dada temperatura e, na água, colocar o corpo cuja temperatura tenha sido previamente medida. A partir deste instante deve-se medir, em intervalos regulares de tempo, a temperatura do sistema. Existem pelo menos duas formas experimentais básicas que utilizam a lei de resfriamento de Newton para a determinação do calor específico de uma substância. Na primeira delas o processo de imersão seguido das leituras de temperaturas é feito duas vezes: uma para um corpo padrão, de calor específico conhecido, e outra para o corpo cuja substância se deseja determinar o calor específico. Esta forma de utilização da lei de resfriamento é detalhada, por exemplo, na Ref. [5] que dispõe, inclusive, de um applet sobre o assunto. Uma outra forma de utilização da lei do resfriamento de Newton procura determinar a temperatura do sistema imediatamente antes da imersão do corpo, e também a temperatura do sistema logo após a migração interna de calor devido à imersão. Neste caso é pressuposto que o arranjo experimental seja tal que as migrações internas de calor devido à imersão possam ser consideradas suficientemente rápidas, de tal forma a se identificar uma "temperatura final de equilíbrio". Identificada esta temperatura, a equação de balanço de calor pode ser utilizada para a determinação do calor específico. Este raciocínio foi utilizado sem muito rigor matemático em [6], [7] e [8]. No experimento descrito em [6] usou-se uma pequena quantidade de alumínio maciço, em comparação com a capacidade calorífica do restante do sistema. Isto provocou uma pequena variação de temperatura devido à imersão do alumínio. A vantagem deste procedimento é ter duas curvas de decaimento com inclinações próximas, o que facilita a estimativa da diferença de temperaturas antes e após a imersão, com um resultado aceitável. Por outro lado, mesmo considerando as medidas de massas como exatas e pequenas incertezas para as temperaturas do sistema, estimadas antes e após a imersão do bloco de alumínio, a precisão experimental não é boa. Naturalmente os autores deixaram claro que a proposta experimental visava ao ensino médio e, ao que parece, o essencial seria apresentar a idéia da alternativa de "não uso" de um calorímetro.

O objetivo do presente artigo é utilizar a técnica da medição de calor específico levando em consideração o contínuo resfriamento do sistema, como em [6], discutir uma forma adequada para a sua realização num curso básico de Física Experimental para o nível superior de ensino e propor um refinamento nas análises dos dados, de forma a se "determinar" e não "estimar" as temperaturas imediatamente antes da imersão e logo após o processo de migração interna de calor devido à imersão.

\section{Planejamento da experiência}

O experimento será planejado para ter um baixo custo, mas assegurando-se a necessária condição de bons resultados. Isto posto, o recipiente escolhido para conter água pode ser uma lata de refrigerante sem o tampo, e o motivo, além do custo, é que o seu material é o mesmo para o qual será determinado o calor específico: o alumínio. Este recipiente normalmente contém $350 \mathrm{ml}$ de refrigerante mas a sua capacidade máxima é de $375 \mathrm{ml}$. O alumínio, propriamente dito, pode ser adquirido em sucata, de preferência na forma de uma vareta que, então, deve ser serrada em pequenos lingotes compatíveis com o tamanho do recipiente.

Como a maioria dos relógios de pulso dispõe de cronômetro, esta é a opção mais econômica para o experimento.

Além de água, praticamente sem custo na quantidade requerida, o experimento necessita, conforme foi mencionado, de alumínio. Como esta proposta experimental irá requerer que as migrações internas de calor sejam muito rápidas, após a imersão do alumínio, a idéia adequada é usar um grande número de pequenas barras dessa substância, aumentando a área de contato alumínio-água. No caso, pode-se usar cerca 30 lingotes de seção reta de aproximadamente $0,7 \mathrm{~cm} \times 0,7$ $\mathrm{cm}$, com comprimento aproximado de $9,0 \mathrm{~cm}$. Essa quantidade é compatível com o volume e a capacidade térmica do restante do sistema, e a razão para o uso dos lingotes será apresentada em detalhes na seção 6 .

Dos materiais necessários, dois deles realmente têm um certo custo, ainda que baixo: o termômetro de vidro a mercúrio $\left(-10\right.$ a $\left.110^{\circ} \mathrm{C}\right)$ e a balança. Neste experimento foi utilizada uma balança de dois pratos (200 g) disponível no KEM [9].

Preferencialmente, o experimento deve ser realizado num horário em que a variação de temperatura ambiente não seja significativa ao longo da coleta de dados.

Pode-se usar o procedimento de pré-aquecer o sistema recipiente/água num fogão comum, depois medir a sua temperatura a cada 1,5 min e, num dado instante, mergulhar os lingotes de alumínio, inicialmente na temperatura ambiente. Após alguns instantes, a temperatura do novo sistema também deve ser medida, por exemplo, a cada $3 \mathrm{~min}$. 
Tais intervalos de tempo são compatíveis com o número de pontos experimentais desejados, considerando-se o tempo previamente estipulado para a duração do experimento, em torno de $1 \mathrm{~h}$, com uma temperatura inicial do sistema por volta de $80^{\circ} \mathrm{C}$ e temperatura ambiente próxima a $25^{\circ} \mathrm{C}$.

\section{Teoria}

Muito embora os procedimentos experimentais requeiram análises teóricas relativamente simples, é bom ressaltar alguns pontos acerca dos fenômenos envolvidos.

\subsection{Lei de resfriamento de Newton}

Embora a expressão que dá nome ao item 3.1 possa soar familiar a estudantes do ciclo básico de cursos superiores relacionados à área de ciências exatas, é surpreendente que praticamente nenhum livro destinado a esse segmento dê ênfase ao tema nos capítulos referentes à termodinâmica. Por isso se justifica uma revisão teórica sobre o assunto. Para tal, seja um sistema em que se possa admitir uma simplificação: a mesma temperatura $T$ em todos os seus pontos. Supondo que tal sistema esteja em um ambiente cuja temperatura seja $T_{a}$, sendo $T_{a}<T$, haverá um fluxo de calor do mais quente para o mais frio. Observações experimentais indicam que a corrente térmica estabelecida, isto é, a quantidade de calor transferida do mais quente para o mais frio por unidade de tempo, $d Q / d t$, é proporcional à diferença de temperaturas $\left(T--T_{a}\right)$ entre o sistema e o ambiente (ver [4], [5] e [10], por exemplo):

$$
\frac{d Q}{d t}=h A\left(T-T_{a}\right)
$$

em que $h$ é uma constante característica de cada sistema e Aé a área de contato entre o sistema e o meio. Para o caso em que o sistema seja considerado um "reservatório finito" de calor e o ambiente, um "reservatório infinito", a temperatura $T$ do sistema varia com o tempo mas a temperatura $T_{a}$ do ambiente não. Lembrando que a transferência de uma quantidade infinitesimal de calor $d Q$ do sistema ao ambiente é igual $a--C d T$, onde $C$ é a capacidade calorífica de tal sistema e $d T$ é a sua variação infinitesimal de temperatura devido à transferência, pode-se reescrever a Eq. (1) da seguinte forma:

$$
\frac{d T}{\left(T-T_{a}\right)}=-(1 / \tau) d t
$$

em que $\tau=C /(h A)$. A Eq. (2) é uma equação diferencial em que a variável do primeiro membro é a temperatura $T$ do sistema enquanto que a do segundo membro é o tempo $t$ de resfriamento. Admitindo que no instante $t=t_{0}$ a temperatura do sistema seja $T_{0}$, e integrando o primeiro membro da Eq. (2) de $T_{0}$ a $T$ e o segundo membro de $t_{0}$ até um instante $t$, ao final tem-se

$$
T=T_{a}+\left(T_{0}-T_{a}\right) e^{-\left(t-t_{0}\right) / \tau} .
$$

A Eq. (3) descreve a forma como um reservatório finito de calor (sistema) é resfriado perdendo calor para um reservatório infinito (meio ambiente), ao longo do tempo.

\subsection{Trocas de calor entre os elementos do sis- tema}

O experimento foi planejado de forma que as migrações internas de calor, após a imersão dos 30 pequenos lingotes de alumínio possam ser consideradas "instantâneas", já que existe uma grande área de contato entre as substâncias envolvidas. Uma análise da validade dessa suposição para o arranjo experimental será feita na seção 6. Assim, durante o curto período de introdução dos lingotes, estimado em 1 ou 2 segundos, pode-se considerar que não haja perda significativa de calor do sistema para o meio ambiente. Neste caso, temse uma massa $M_{R}$ do recipiente de alumínio e uma massa $M$ de água numa temperatura $T_{q}$. Uma massa $M_{A l}$ de alumínio na temperatura ambiente $T_{a}$ é introduzida no sistema e, após as trocas internas de calor, a temperatura do sistema passa a ser $T_{f}$. Simbolizando o calor específico da água por $c_{a g}$, a expressão para o calor específico do alumínio é:

$$
c=\frac{M\left(T_{q}-T_{f}\right)}{M_{A l}\left(T_{f}-T_{a}\right)-M_{R}\left(T_{q}-T_{f}\right)} c_{a g} .
$$

Passemos às medidas realizadas.

\section{Medidas realizadas}

As medições efetuadas envolveram não só os valores médios, em si, mas também as incertezas das medidas, levando-se em consideração o instrumento utilizado.

\subsection{Medidas de massa}

No experimento foi utilizada uma balança de dois pratos. Para eliminar erros devido à assimetria da barra da balança com relação ao ponto de apoio, o corpo foi colocado em cada um dos dois pratos, seguindo-se a medição. O melhor valor, então, é dado pela média geométrica dos valores medidos [11] (Pág. 76) e [12] (Pág. 37). Às massas padronizadas foi associada uma incerteza de $2 \%$ do valor final e, assim, foram obtidos:

$$
\begin{aligned}
\text { Massa de água } M & =(266 \pm 5) \mathrm{g} \\
\text { Massa do recipiente } M_{R} & =(11,60 \pm 0,23) \mathrm{g} \\
\text { Massa de alumínio } M_{A l} & =(278 \pm 6) \mathrm{g}
\end{aligned}
$$

\subsection{Medições diretas de temperaturas}

A temperatura ambiente $T_{a}$ foi medida no início e no fim do experimento, não havendo diferença detectável nos valores obtidos:

$$
T_{a}=(25,0 \pm 0,5)^{0} \mathrm{C} .
$$

As temperaturas $T_{q}$ (antes da imersão dos lingotes de alumínio) e $T_{f}$ (imediatamente após à imersão dos lingotes) 
serão obtidas por ajustes da Eq. (3) aos pontos experimentais das Tabelas 1 e 2.

$\mathrm{O}$ recipiente com água quente foi colocado sobre uma bancada, começando-se o processo de resfriamento, que gerou os dados da Tabela 1 .

Tabela 1. Decaimento da temperatura $T$ ao longo do tempo $t$ antes da imersão do alumínio (região I)

\begin{tabular}{|l|l|l|l|l|l|l|l|}
\hline & 1 & 2 & 3 & 4 & 5 & 6 & 7 \\
\hline$t(\min )$ & 0,0 & 1,5 & 3,0 & 4,5 & 6,0 & 7,5 & 9,0 \\
\hline$T\left({ }^{0} \mathrm{C}\right)$ & 82,0 & 79,5 & 77,0 & 74,5 & 72,0 & 70,0 & 68,0 \\
\hline
\end{tabular}

Aos 9 min e $20 \mathrm{~s}$, isto é, no instante $t=9,33 \mathrm{~min}$ os 30 lingotes à temperatura ambiente foram introduzidos, de uma só vez, no recipiente com água quente. A partir do instante $t=12$ min o processo de medição da temperatura foi reiniciado, com uma freqüência de 3 em 3 min. Os resultados são dados na Tabela 2.

Tabela 2. Decaimento da temperatura $T$ ao longo do tempo $t$ após a imersão do alumínio (região II)

\begin{tabular}{|l|l|l|l|l|l|l|l|l|l|l|l|l|}
\hline & 1 & 2 & 3 & 4 & 5 & 6 & 7 & 8 & 9 & 10 & 11 & 12 \\
\hline$t(\min )$ & 12,0 & 15,0 & 18,0 & 21,0 & 24,0 & 27,0 & 30,0 & 33,0 & 36,0 & 39,0 & 42,0 & 45,0 \\
\hline$T\left({ }^{0} \mathrm{C}\right)$ & 59,0 & 57,5 & 56,5 & 55,5 & 54,5 & 53,0 & 52,0 & 51,0 & 50,2 & 49,5 & 48,5 & 47,5 \\
\hline
\end{tabular}

\begin{tabular}{|l|l|l|l|l|}
\hline 13 & 14 & 15 & 16 & 17 \\
\hline 48,0 & 51,0 & 54,0 & 57,0 & 60,0 \\
\hline 46,8 & 46,0 & 45,2 & 44,5 & 43,6 \\
\hline
\end{tabular}

\section{Análise dos dados e determinação do calor específico do alumínio}

Para se ter uma idéia concreta sobre as duas fases distintas do resfriamento (antes e após a imersão dos lingotes de alumínio) basta observar o esboço gráfico de $T \times t$ com todos os pontos experimentais em um único sistema de eixos, conforme mostra a Fig. 1.

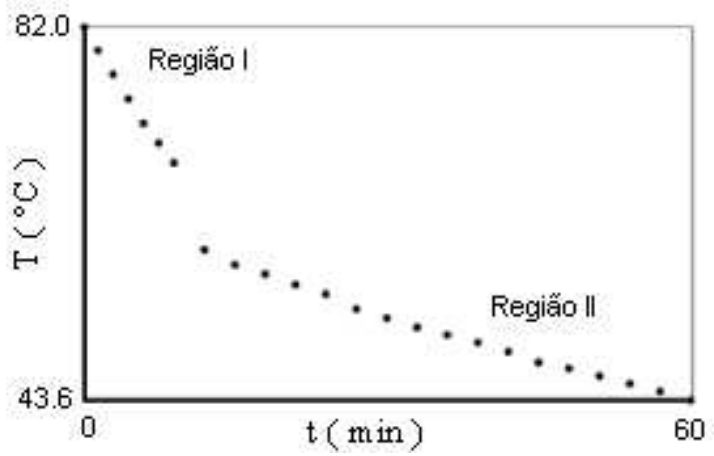

Figura 1. Resfriamento do sistema antes da imersão do alumínio (região I) e depois da imersão (região II).

\subsection{Determinação da temperatura $T_{q}$}

Um aspecto importante a ser observado no refinamento proposto neste artigo diz respeito à determinação das temperaturas do sistema imediatamente antes da imersão dos lingotes de alumínio e logo após às migrações internas de calor. Isto será feito ajustando a própria função que descreve a lei de resfriamento (não-linear nos parâmetros) aos dados experimentais, e não um polinômio, como a intuição pode sugerir num primeiro momento, o que corresponderia a uma solução simplificada, que poderia até gerar bons resultados, mas sem a descrição direta da Física envolvida. O procedimento a ser usado possibilitará fazer uma comparação dos parâmetros obtidos experimentalmente com aqueles previstos pela teoria, o que constituirá em um dos indicadores da consistência do experimento realizado.

A determinação da temperatura $T_{q}$ do sistema (imediatamente antes da imersão) pode ser feita reescrevendo a Eq. (3) na forma

$$
T=A 1+A 2 e^{A 3\left(t-t_{0}\right)},
$$

e ajustando essa função não-linear nos parâmetros aos dados da Tabela 1, observando que, nesta região, $t_{0}=0$. O software utilizado foi o LABFit [13] porque tal software informa não só os valores médios e os desvios dos parâmetros, mas também as covariâncias entre tais parâmetros; dadas através de uma matriz $M$, chamada de matriz das covariâncias. $\mathrm{O}$ conhecimento da matriz das covariâncias possibilita fazer extrapolações obtendo não só o valor médio da função ajustada, mas também o desvio padrão deste valor médio, o que envolve propagação de erros. Detalhes sobre o assunto "propagação de erros" podem ser obtidos nas Ref. [11] (Cap. 8), [12] (Cap. 4), [14] e [15]. Já detalhes sobre a aplicação de propagação de erros especificamente em ajuste de curvas podem ser encontrados em [12] (Seções 6.6, 6.7 e 6.8). Voltando ao ajuste da Eq. (7) aos dados da Tabela 1, os resultados completos para tal ajuste são os seguintes [16]: 
- $\mathrm{A} 1=26,9732 \ldots . \quad \sigma_{A 1}=11,4537 \ldots \ldots$

- $\mathrm{A} 2=55,10968 \ldots . \quad \sigma_{A 2}=11,3877 \ldots \ldots$

- $\mathrm{A} 3=-0,032983 \ldots . \quad \sigma_{A 3}=0,0079235 \ldots$.

$$
M=\left|\begin{array}{ccc}
0,131188 E+03 & -0,130428 E+03 & -0,906708 E-01 \\
-0,130428 E+03 & 0,129681 E+03 & 0,901207 E-01 \\
-0,906708 E-01 & 0,901207 E-01 & 0,627833 E-04
\end{array}\right|
$$

A representação gráfica da função ajustada, com uma pequena extrapolação até $t=9,33 \mathrm{~min}$, pode ser vista na Fig. 2.

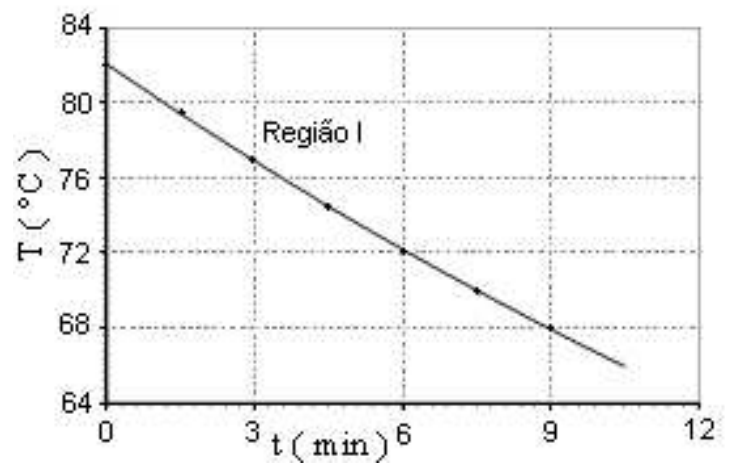

Figura 2. Decaimento da temperatura $T$ ao longo do tempo $t$ antes da imersão do alumínio (região I).

- $\mathrm{A} 1=24,5023 \ldots$

- $\mathrm{A} 2=34,4082 \ldots$

- $\mathrm{A} 3=-0,012122 \ldots$
O valor obtido para a Eq. (7) em $t=9,33$ min, utilizando a fórmula geral de propagação de erros e os resultados encontrados em (8), é o seguinte:

$$
T_{q}=(67,48 \pm 0,36)^{0} \mathrm{C},
$$

com $95,4 \%$ de confiança. Devido ao número de pontos, deve-se observar que o valor originalmente obtido para a incerteza de $T_{q}$ foi multiplicado pelo fator 2,87 (supondo-se uma distribuição gaussiana dos pontos em torno da função ajustada) para que o intervalo dado em (9) tenha a confiança de $95,4 \%$ de conter o valor verdadeiro.

\subsection{Determinação da temperatura $T_{F}$}

Observando que na segunda região $t_{0}=12,0 \mathrm{~min}$, e ajustando a Eq. (7) aos dados da Tabela 2 (depois da imersão), obtém-se os seguintes resultados [16]:

$$
\begin{aligned}
& \sigma_{A 1}=2,5128 \ldots . \\
& \sigma_{A 2}=2,4509 \ldots . \\
& \sigma_{A 3}=0,001169 \ldots .
\end{aligned}
$$

$$
M=\left|\begin{array}{llc}
0,631454 E+01 & -0,615588 E+01 & -0,292925 E-02 \\
-0,615588 E+01 & 0,600699 E+01 & 0,284996 E-02 \\
-0,292925 E-02 & 0,284996 E-02 & 0,136761 E-05
\end{array}\right|
$$

O gráfico da função ajustada pode ser visto na Fig. 3, que mostra também uma pequena extrapolação até $t=9,33$ $\min$.

Neste novo ajuste, o valor da temperatura do sistema para $t=9,33$ min é:

$$
T_{f}=(60,04 \pm 0,28)^{0} \mathrm{C},
$$

com $95,4 \%$ de confiança. Devido ao número de pontos, deve-se observar que o valor originalmente obtido para a incerteza de $T_{f}$ foi multiplicado pelo fator 2,18 (supondose uma distribuição gaussiana dos pontos em torno da função ajustada) para que o intervalo dado em (11) tenha a confiança de $95,4 \%$ de conter o valor verdadeiro.

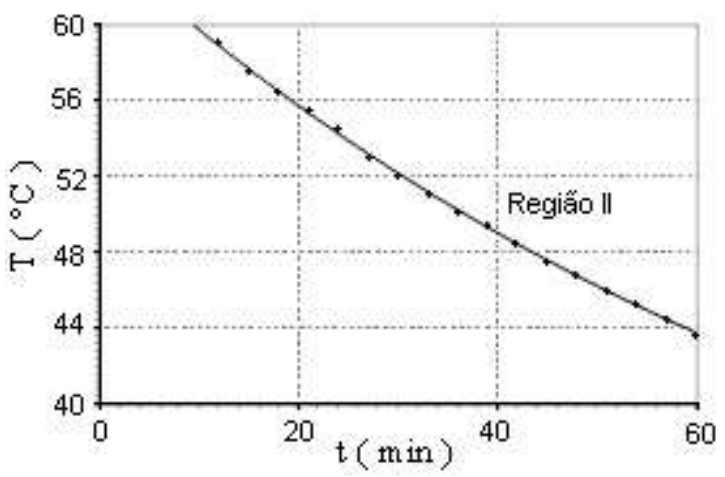

Figura 3. Decaimento da temperatura $T$ ao longo do tempo $t$ após a imersão do alumínio (região II). 


\subsection{Cálculo do calor específico}

Com as medidas indicadas em (5) e em (6), e com as temperaturas determinadas em (9) e em (11), pode-se usar, novamente, o software LABFit, que dispõe de uma opção para o cálculo do valor médio e do errro propagado de expressões tal como aquela da Eq. (4). Neste cálculo será usado, para o calor específico da água na temperatura de $15^{\circ} \mathrm{C}$, o valor $c_{a g}=1 \mathrm{cal} /\left(\mathrm{g}^{\circ} \mathrm{C}\right)$ [17]. Este é um valor definido e, portanto, isento de erros (incerteza igual a zero). Então, através da Eq. (4) obtém-se:

$$
c=(0,205 \pm 0,015) \mathrm{cal} /\left(\mathrm{g}^{0} \mathrm{C}\right) .
$$

Há que se observar, nesse último cálculo, que os parâmetros da Eq. (4) podem ser considerados independentes e que, portanto, as covariâncias entre parâmetros distintos são iguais a zero. Apenas como informação adicional, apesar de pequenas discrepâncias na literatura, um valor de referência para o calor específico do alumínio é dado assim [17]:

$$
c=0,214 \mathrm{cal} /\left(\mathrm{g}^{o} \mathrm{C}\right) .
$$

Devido à simplicidade dos procedimentos e materiais experimentais, incluindo-se aí os instrumentos de medidas, não deve ser considerado como muito significativo o fato do calor específico do alumínio dado em (13) corresponder à temperatura de $20^{\circ} \mathrm{C}$ e o resultado obtido em (12) ter envolvido o intervalo de 60,04 a $67,48^{\circ} \mathrm{C}$, além do valor do calor específico da água ser referente a $15^{\circ} \mathrm{C}$. Dentre outros aspectos que não foram levados em conta nos cálculos pode-se destacar a evaporação da água no recipiente durante o resfriamento, erros sistemáticos introduzidos pelos instrumentos de medida e também o tipo de alumínio utilizado (pureza e porosidade, por exemplo).

\section{Discussões e Conclusões}

É interessante como a análise de alguns resultados pode indicar a consistência do experimento realizado. A Fig. 1 mostra duas regiões distintas de $T \times t$ para as quais, supostamente, a lei que descreve o fenômeno observado é a lei de resfriamento de Newton. Se isso for correto, os parâmetros A1, A2 e A3 da Eq. (7), obtidos por ajuste de curvas, devem conter informações importantes que, num primeiro momento, podem escapar. Como na primeira região foram feitas poucas medidas, o que significa um baixo número de parâmetros para o ajuste (apenas quatro), o foco da análise de alguns resultados será a região 2 . O parâmetro A3 tem a interpretação usual relacionada a fenômenos de decaimento descritos por funções exponenciais: inverso da constante de tempo. O interessante, entretanto, são as interpretações para os parâmetros A1 e A2. Comparando a Eq. (3) com a Eq. (7), percebe-se que o parâmetro A1 da Eq. (7) (função de ajuste) vem a ser a temperatura ambiente $T_{a}$ da Eq. (3) (lei de resfriamento). $\mathrm{O}$ valor médio do parâmetro $\mathrm{A} 1$ obtido no ajuste de curvas para a região II foi $24,5^{\circ} \mathrm{C}$, enquanto que a medição direta da temperatura ambiente indicou $T_{a}=$ $25,0^{\circ} \mathrm{C}$. Já o valor médio do parâmetro A2 é, teoricamente, igual à diferença entre a temperatura $T_{0}$ do início do resfriamento (igual a $59,0^{\circ} \mathrm{C}$ em $t_{0}=12,0 \mathrm{~min}$, na região II) e a temperatura ambiente $T_{a}$ (igual a $25,0^{\circ} \mathrm{C}$ ). Na região II, o valor obtido para A2 por ajuste de curvas foi $34,4^{\circ} \mathrm{C}$, enquanto que, por medição direta, $T_{0}--T_{a}=34,0^{\circ} \mathrm{C}$. As discussões anteriores sobre os parâmetros A1 e A2 mostram uma grande coerência entre os resultados experimentais e a previsão teórica dada pela lei de resfriamento.

Quanto à determinação do calor específico, em si, um aspecto importante nos procedimentos experimentais foi o fato de se fragmentar a peça de alumínio em pequenos pedaços, o que contribuiu para uma rápida migração interna de calor, após a imersão. É isto que possibilita determinar as temperaturas $T_{q}$ e $T_{f}$ supondo que as duas temperaturas possam ser calculadas por extrapolação, ambas, "no mesmo instante".

Ao planejar o experimento, não foi atribuída muita importância ao fato de que as inclinações das curvas nas duas regiões fossem tão significativamente diferentes. Naturalmente, neste caso, se as migrações internas de calor não forem "praticamente instantâneas" a diferença $\left(T_{q}-T_{f}\right)$, obtida no experimento, seria afetada por erros que inviabilizariam a credibilidade do resultado. Na verdade, esta diferença significativa nas inclinações das curvas nas duas regiões foi intencionalmente provocada, porque se os resultados em condições tão adversas forem satisfatórios, a validade do refinamento proposto neste artigo para o experimento fica completamente assegurada.

Deve-se observar que o único instrumento "sofisticado" utilizado em todo o processo foi o software de análise de dados. Todo o material experimental é de custo reduzido e, mesmo assim, os resultados obtidos foram muito favoráveis, levando-se em consideração o propósito educacional do experimento. A precisão do resultado final foi de $6,5 \%$ enquanto que a comparação do valor médio obtido para o calor específico, dado em (12), com o valor de referência, dado em (13), indica um erro de 4,2\%. Tais percentuais são indicadores de que o refinamento proposto é útil quando não se dispõe de um bom calorímetro. Por outro lado, uma análise mais atenta do resultado para o calor específico indica que pode ter havido erros sistemáticos que mereçam atenção, já que o intervalo de confiança do valor obtido, dado em (12), contém o valor de referência, dado em (13), mas um pouco próximo do limite superior.

Para reforçar a validade dos procedimentos propostos, pode-se repetir o experimento com a lata de refrigerante inserida em isopor, o que diminuiria as inclinações das duas regiões mostradas na Fig. 1, e tal procedimento deve melhorar de forma significativa os resultados obtidos. Isso fica como uma sugestão para trabalhos futuros.

Um ponto que merece reflexão é o fato de ter sido assumido que as migrações internas de calor na imersão do material possam ser consideradas instantâneas, num tempo $t$. Apesar da fragmentação da vareta de alumínio em muitos lingotes pequenos e finos, foi feito um estudo considerando que as migrações tivessem demorado um certo tempo $\Delta t$. Nesse caso, o valor de $T_{q}(t)$ permanece o mesmo determinado no instante $t=9,33 \mathrm{~min}$. Mas $T_{f}(t+\Delta t)$ seria menor que o valor obtido e, portanto, deveria ser determinado, com o mesmo procedimento já utilizado, que é a extrapolação 
da função de resfriamento. Para essa nova situação, entretanto, nem todo calor perdido pelo sistema recipiente/água será transferido para os lingotes de alumínio. Uma pequena parte, correspondente à variação da temperatura do sistema recipiente/água de $T_{q}(t)$ até $T_{q}(t+\Delta t)$ seria perdida para o ambiente. Para se ter uma idéia do possível intervalo de tempo $\Delta t$ necessário para o equilíbrio térmico, foi feita a seguinte medida: o intervalo de tempo para um termômetro de vidro a mercúrio, inicialmente, a $25^{\circ} \mathrm{C}$, indicar a temperatura de água fervente. Tal intervalo de tempo é da ordem de $10 \mathrm{~s}$. Então, foram feitas duas simulações com os dados obtidos no experimento, uma supondo $\Delta t=5 \mathrm{~s}$ e outra com $\Delta t$ $=10 \mathrm{~s}$. Os resultados das simulações mostraram que o valor determinado para o calor específico do alumínio não sofreu alterações muito significativas (apesar de um suave declínio deste valor) e isso reforça o que foi assumido: migrações instantâneas de calor no processo de imersão dos lingotes.

Uma conclusão final: deve-se observar que o refinamento proposto neste artigo pode ser estendido para a realização do experimento com um calorímetro. Neste caso, as duas regiões da Fig. 1 seriam descritas por linhas com inclinações tendendo a zero, e a função de ajuste adequada aos pontos seria, então, a função constante $(T=\mathrm{A} 1)$. Isto é compatível com a lei de resfriamento na qual o valor de $1 / \tau$ tenda para zero, o que resulta em $T=T_{0}$. Nestas circunstâncias, o resultado do ajuste de cada curva recairia no caso do tratamento de leituras de uma mesma quantidade. A temperatura de cada região seria dada pelo valor médio da série de leituras efetuadas (valor médio da função constante $T=\mathrm{A} 1$ ) e a sua incerteza seria dada pelo desvio padrão deste valor médio $\left(\sigma_{A 1}\right)$.

Os autores Diogo e Cleiton D.P.S. e Silva são respectivamente bolsistas do PIBIC (CNPq) na UFCG e da FAPESP no Mestrado em Engenharia Eletrônica do ITA.

\section{Referências}

[1] Albuquerque, W.V et al., Manual de Laboratório de Física Ed. McGraw-Hill do Brasil, São Paulo, SP, $1^{\text {a }}$ Edição (1980),
Pág. 121

[2] Tipler, P. A., Física, Editora Guanabara Dois S. A. Rio de Janeiro, RJ, Vol. 1, $1^{a}$ Edição, (1978), Pág. 445, Prob. 5

[3] Weltner, K. e Miranda, P., Rev. Bras. Ens. Fís., 20, 301 (1998)

[4] Vuolo, J. H. e Furukawa, C. H., Rev. Bras. Ens. Fís., 17, 140 (1995)

[5] Texto online, em http://www.sc.ehu.es/sbweb/fisica /estadistica/otros/enfriamiento/enfriamiento.htm

[6] Mattos, C. R. e Gaspar, A., Rev. Bras. Ens. Fís., 25, 45 (2003)

[7] Mattos, C. R. e Gaspar, A, PDF disponível online, http://webpages.ull.es/users/apice/pdf/121-063.pdf

[8] Mattos, C. R. e Gaspar, A., Phys. Teach., 40, 415 (2002)

[9] KEM - Kit para Experiências de Mecânica, info online, http://www.extensao.hpg.com.br/kits/kits.html

[10] Sartorelli, J. C. et al., Rev. Bras. Ens. Fís., 21, 116 (1999)

[11] Vuolo, J. H., Fundamentos da Teoria dos Erros, Ed. Edgard Blücher Ltda São Paulo, SP, $1^{a}$ Edição, (1992)

[12] Silva, W. P. e Silva, C. M. D. P. S., Tratamento de Dados Experimentais UFPB Editora Universitária, João Pessoa, PB, $2^{a}$ Edição, (1998)

[13] LAB Fit Curve Fitting Software, online, download disponível em http://www.angelfire.com/rnb/labfit

[14] Taylor, J. R., An Introduction to Error Analysis, University Science Books, Suasalito, CA Second Edition (1997), Chapter 9

[15] Helene, O. A. M. e Vanin, V. R., Tratamento Estatístico de Dados em Física Experimental, Editora Edgard Blücher Ltda, São Paulo, SP, $1^{a}$ Edição (1981), Cap. IV Seção f

[16] Em regressão não-linear os parâmetros são determinados através de correções iterativas dos valores iniciais estipulados pelo usuário. Nos dois ajustes deste artigo os valores iniciais dos parâmetros foram: $\mathrm{A} 10=1, \mathrm{~A} 20=1$ e A30 $=0$

[17] Eisberg, R. M. e Lerner, L. S., Física Fundamentos e Aplicações, Ed. McGraw-Hill do Brasil, São Paulo, SP, Vol. 2, $1^{a}$ Edição, (1982), Pág. 399 